Annales Geophysicae (2002) 20: 967-979 (c) European Geophysical Society 2002

\title{
Evidence for acceleration of outer zone electrons to relativistic energies by whistler mode chorus
}

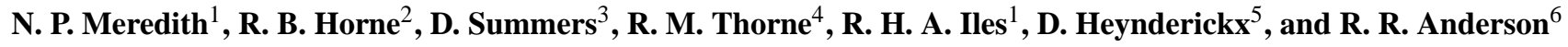 \\ ${ }^{1}$ Mullard Space Science Laboratory, University College London, Holmbury St Mary, Dorking, Surrey, UK \\ ${ }^{2}$ British Antarctic Survey, Natural Environment Research Council, Madingley Road, Cambridge, UK \\ ${ }^{3}$ Department of Mathematics and Statistics, Memorial University of Newfoundland, St. John's, Newfoundland, Canada \\ ${ }^{4}$ Department of Atmospheric Sciences, University of California, Los Angeles, USA \\ ${ }^{5}$ Belgian Institute for Space Aeronomy, Ringlaan 3, Brussels, Belgium \\ ${ }^{6}$ Department of Physics and Astronomy, The University of Iowa, Iowa City, Iowa, USA
}

Received: 27 September 2001 - Revised: 18 March 2002 - Accepted: 20 March 2002

\begin{abstract}
We use plasma wave and electron data from the Combined Release and Radiation Effects Satellite (CRRES) to investigate the viability of a local stochastic electron acceleration mechanism to relativistic energies driven by gyroresonant interactions with whistler mode chorus. In particular, we examine the temporal evolution of the spectral response of the electrons and the waves during the 9 October 1990 geomagnetic storm. The observed hardening of the electron energy spectra over about 3 days in the recovery phase is coincident with prolonged substorm activity, as monitored by the $A_{E}$ index and enhanced levels of whistler mode chorus waves. The observed spectral hardening is observed to take place over a range of energies appropriate to the resonant energies associated with Doppler-shifted cyclotron resonance, as supported by the construction of realistic resonance curves and resonant diffusion surfaces. Furthermore, we show that the observed spectral hardening is not consistent with energy-independent radial diffusion models. These results provide strong circumstantial evidence for a local stochastic acceleration mechanism, involving the energisation of a seed population of electrons with energies of the order of a few hundred $\mathrm{keV}$ to relativistic energies, driven by wave-particle interactions involving whistler mode chorus. The results suggest that this mechanism contributes to the reformation of the relativistic outer zone population during geomagnetic storms, and is most effective when the recovery phase is characterised by prolonged substorm activity. An additional significant result of this paper is that we demonstrate that the lower energy part of the storm-time electron distribution is in steady-state balance, in accordance with the Kennel and Petschek (1966) theory of limited stably-trapped particle fluxes.
\end{abstract}

Key words. Magnetospheric physics (storms and sub-

Correspondence to: N. P. Meredith (npm@mssl.ucl.ac.uk) storms, energetic particles, trapped) - Space plasma physics (wave-particle interactions)

\section{Introduction}

In a "typical" geomagnetic storm, electrons are accelerated during the recovery phase to energies $\geq 1 \mathrm{MeV}$ in the outer radiation zone $(3<L<7)$. The problem of explaining this phenomenon is attracting increasing interest among magnetospheric physicists. The generation of such relativistic electrons constitutes a potential hazard to satellite instruments (e.g. Baker, 1996; Baker et al., 1997) and humans in space. The equatorial region near geosynchronous location at $L=6.6$ is especially significant since it is the operating zone of many orbiting satellites. Understanding the generation mechanisms of the relativistic electrons is an important, challenging problem, and predicting their appearance has become a key element in the science of space weather.

While the generation of storm-time relativistic electrons has attracted increasing theoretical effort, a definitive theory has yet to emerge (see, for example, Li and Temerin, 2001). Radial diffusion (Schulz and Lanzerotti, 1974) has been examined by various authors as a possible generator of $\mathrm{MeV}$ electrons in the inner magnetosphere. Radial diffusion conserves the first two adiabatic invariants but breaks the third, and may result in significant electron energisation when the electron phase space density (at constant adiabatic invariants) increases with radial distance. Global recirculation processes involving radial diffusion have been proposed to generate relativistic electrons by Baker et al. (1989) and Fujimoto and Nishida (1990). Beutier and Boscher (1995) have developed an extensive computer code (Salammbô) to solve a threedimensional phase space diffusion equation for the electron radiation belt population. Kim et al. (2000) and Fok et al. 
(2001) modelled the inward radial diffusion of plasma sheet electrons and their injection into the outer zone during a dipolarisation event.

Ultra low frequency (ULF) waves have also been examined as a potential generator of relativistic electrons during storms. Mathie and Mann (2000) and O'Brien et al. (2001) established a correlation between relativistic electron events at $L=6.6$ and prolonged ULF wave activity during the storm recovery. Liu et al. (1999) proposed an acceleration mechanism involving magnetic pumping by ULF waves, while Hudson et al. (2000) and Elkington et al. (1999) proposed a mechanism involving drift resonant acceleration by ULF waves associated with inward radial transport. Drift resonant acceleration with ULF waves also conserves the first two adiabatic invariants but breaks the third and is one of the drivers for radial diffusion.

Models which are based purely on radial diffusion have shortcomings. Brautigam and Albert (2000) carried out a radial diffusion analysis of the outer zone electrons during the 9 October 1990 geomagnetic storm and showed that the higher energy electrons exhibited a decrease in phase space density with increasing $L$, which is inconsistent with an inward radial diffusion source. Furthermore, during the recovery phase, the radial profile of the phase space density exhibited a local maximum near $L=4$, inconsistent with standard radial diffusion models and indicative of a local acceleration source.

There is mounting evidence that an internal in situ mechanism in the inner magnetosphere causes strong enhancements in the outer belt $\mathrm{MeV}$ electrons during magnetic storms (e.g. Baker et al., 1996; Blake et al., 1998; Li et al., 1999; Brautigam and Albert, 2000). A possible mechanism for accelerating electrons internally in the inner magnetosphere is that of stochastic acceleration during cyclotron resonant interaction of electrons with high frequency plasma waves, which violates the first, and hence, all three of the adiabatic invariants. Horne and Thorne (1998) showed that there are five different wave modes that can resonate with electrons in the energy range from $100 \mathrm{keV}$ to a few $\mathrm{MeV}$ in different regions of the Earth's magnetosphere. One of the most important is the whistler mode wave. Stochastic acceleration of electrons by whistler mode waves has been discussed by Summers et al. (1998), Roth et al. (1999) and Summers and Ma (2000). Summers et al. (1998) extended the non-relativistic theory of wave-particle gyroresonant diffusion to relativistic plasmas, and showed that whistlers could efficiently accelerate electrons from energies $\sim 100 \mathrm{keV}$ to above $1 \mathrm{MeV}$ in the region outside of the plasmapause during the storm recovery. Summers and Ma (2000) developed quantitatively the model proposed by Summers et al. (1998) and formulated a kinetic (Fokker-Planck) equation for the electron energy distribution. Steady-state solutions of the equation were constructed using observed electron data at $L=6.6$, and the model solutions showed that stochastic acceleration by whistler mode waves is a viable mechanism for producing relativistic electrons (on a time scale of a few days) for storms possessing a long-lasting recovery phase with sustained whistler mode wave activity.

Observations from the Combined Release and Radiation Effects Satellite (CRRES) provide an ideal data base for studying the role of wave-particle interactions in the generation of relativistic electrons during storms. In this paper, we examine electron and wave data associated with an electron acceleration event during the 9 October 1990 geomagnetic storm. A prime objective is to demonstrate that the observed hardening of the electron energy spectra over about 3 days in the recovery phase is consistent with energisation by whistler mode waves. The consistency is established on the basis of enhanced whistler mode wave power during the recovery phase, and also on the grounds that the spectral hardening takes place over a range of energies appropriate for the resonant energies associated with Doppler-shifted cyclotron resonance. An additional significant result of this paper is that we demonstrate that the lower energy part $(E \leq 500 \mathrm{keV})$ of the storm-time electron distribution is in steady-state balance, in accordance with the Kennel and Petschek (1966) theory of limiting stably-trapped particle fluxes. In the following section we present a brief account of the CRRES spacecraft and associated electron and wave instruments employed in the present study. The data analysis techniques are briefly discussed in Sect. 3. In Sect. 4, we present and discuss the main results of the paper, and in Sect. 5, we present our conclusions.

\section{Instrumentation}

CRRES is particularly well suited to studies of wave-particle interactions in the radiation belts both because of its orbit and sophisticated suite of wave and particle instruments. The spacecraft was launched on 25 July 1990, and operated in a highly elliptical geosynchronous transfer orbit with a perigee of $305 \mathrm{~km}$, an apogee of $35768 \mathrm{~km}$ and an inclination of $18^{\circ}$. The orbital period was approximately $10 \mathrm{~h}$, and the initial apogee was at a magnetic local time of 08:00 MLT. The satellite swept through the heart of the radiation belts approximately 5 times per day on average, providing good coverage of this important region for almost 15 months.

The electron data used in this study were collected by the Medium Electrons A (MEA) experiment. This instrument, which used momentum analysis in a solenoidal field, had 17 energy channels ranging from $153 \mathrm{keV}$ to $1.582 \mathrm{MeV}$ (Vampola et al., 1992). The full field of view, coupled with the angular scan of $6^{\circ}$ which occurred during the $0.512 \mathrm{~s}$ data accumulation period, resulted in a total acceptance angle of $8^{\circ}-18^{\circ}$, depending on the channel.

The wave data used in this study were provided by the University of Iowa plasma wave experiment. This experiment provided measurements of electric fields from $5.6 \mathrm{~Hz}$ to $400 \mathrm{kHz}$, using a $100 \mathrm{~m}$ tip-to-tip long wire antenna, and magnetic fields from $5.6 \mathrm{~Hz}$ to $10 \mathrm{kHz}$, using a search coil magnetometer, with a dynamic range covering a factor of at least $10^{5}$ in amplitude (Anderson et al., 1992). The electric field detector was thus able to detect waves from below 
the lower hybrid resonance frequency $\left(f_{l h r}\right)$ to well above the upper hybrid resonance frequency $\left(f_{u h r}\right)$ for a large fraction of each orbit. The sweep frequency receiver covered the frequency range from $100 \mathrm{~Hz}$ to $400 \mathrm{kHz}$ in four bands with 32 logarithmically spaced steps per band with the fractional step separation, $d f / f$, being about $6.7 \%$ across the entire frequency range. Band $1(100$ to $810 \mathrm{~Hz})$ was sampled at one step per second with a complete cycle time of $32.768 \mathrm{~s}$. Band $2(810 \mathrm{~Hz}$ to $6.4 \mathrm{kHz})$ was sampled at two steps per second with a complete cycle time of $16.384 \mathrm{~s}$. Band 3 (6.4 to $51.7 \mathrm{kHz})$ and band $4(51.7$ to $400 \mathrm{kHz})$ were each sampled 4 times per second, with complete cycling times of $8.192 \mathrm{~s}$. The nominal band widths in each of the four bands were $7 \mathrm{~Hz}, 56 \mathrm{~Hz}, 448 \mathrm{~Hz}$, and $3.6 \mathrm{kHz}$, respectively.

\section{Data analysis}

\subsection{Electrons}

The differential number flux, $J(E, \alpha)$, as a function of energy, $E$, and pitch angle, $\alpha$, was initially rebinned as a function of energy (17 energy channels), pitch angle (in steps of $5^{\circ}$ ), half-orbit (outbound and inbound) and $L$ in steps of $0.1 \mathrm{~L}$. These results were then stored together with the time in UT, magnetic latitude $\left(\lambda_{m}\right)$, magnetic local time (MLT), and time spent in each bin. The perpendicular flux, $J_{\perp}(E)$, at a given energy in a given $L$ bin, was defined as being equal to the average differential number flux in the pitch angle range $85^{\circ}<\alpha<95^{\circ}$.

The differential number flux, $J(E, \alpha)$, was also converted to phase space density in momentum space, $f\left(p_{\perp} / m_{e} c, p_{\|} / m_{e} c\right)$, to investigate the direction of diffusion at a given point in momentum space, driven by gyroresonant interactions with whistler mode waves. This conversion was achieved using the following relativistic relations:

$f=\frac{J}{p^{2}}$

$\frac{p_{\perp}}{m_{e} c}=\frac{1}{E_{0}} \sqrt{E\left(E+2 E_{0}\right)} \sin \alpha$

$\frac{p_{\|}}{m_{e} c}=\frac{1}{E_{0}} \sqrt{E\left(E+2 E_{0}\right)} \cos \alpha$,

where $E_{0}$ is the rest energy of the electron $(0.511 \mathrm{MeV})$.

\subsection{Whistler mode chorus}

The wave data were initially corrected for the instrumental background response, and smoothed using a running 3minute average to take out the beating effects due to differences in the sampling and the spin rate. Spurious data points, data spikes and periods of instrumental downtime were flagged and ignored in the analysis. Whistler mode chorus is commonly observed in the range $0.1 f_{c e}<f<0.8 f_{c e}$ (Koons and Roeder, 1990). Therefore, we initially determined the wave spectral intensity as a function of relative frequency, $x=f / f_{c e}$, in the range $0.02<x<1$ at an $8.192 \mathrm{~s}$ time resolution corresponding to the fastest sweep of the Sweep Frequency Receiver. Here the electron gyrofrequency, $f_{c e}$, was determined directly from the fluxgate magnetometer instrument on board the spacecraft (Singer et al., 1992). The resulting spectral intensities were then averaged into bins of size $x=0.02$ as a function of half-orbit (outbound and inbound) and $L$ in steps of $0.1 L$.

The ratio of the electron plasma frequency to the electron gyrofrequency is an important parameter in the study of gyroresonant wave-particle interactions involving whistler mode chorus (Summers et al., 1998). This ratio, which determines the shape of the resonance curves and resonant diffusion curves, requires knowledge of the electron plasma frequency. This characteristic frequency may be estimated from the plasma wave spectra. Inside the plasmasphere, emissions at the upper hybrid frequency, $f_{u h r}$, are usually well defined and the electron plasma frequency, $f_{p e}$, is estimated from measurements of $f_{u h r}$, using the relationship $f_{p e}^{2}=f_{u h r}^{2}-f_{c e}^{2}$. However, the upper hybrid frequency is not so well defined beyond the plasmapause. In this region, the electron plasma frequency is estimated from the lower frequency limit of the electromagnetic continuum radiation, which is taken to be a plasma wave cutoff at the plasma frequency (Gurnett and Shaw, 1973). The ratio $f_{p e} / f_{c e}$ was determined for each of the fastest sweeps of the Sweep Frequency Receiver at an $8.192 \mathrm{~s}$ time resolution, and then rebinned as a function of half-orbit (outbound and inbound) and $L$ in steps of $0.1 L$.

\section{Results and discussion}

Relativistic electron enhancements have been correlated with the presence of high speed solar wind streams and southward IMF during the recovery phase of geomagnetic storms (e.g. Iles et al., 2002), suggesting that substorm activity may be important. Meredith et al. (2001) performed a detailed statistical analysis of the plasma wave data from the CRRES spacecraft, and showed that outside of the plasmapause the chorus activity was largely substorm-dependent and all chorus emissions were enhanced when the substorm activity was enhanced. Meredith et al. (2002) then analysed three case studies looking for evidence of enhanced substorm activity and whistler mode chorus associated with electron acceleration to relativistic energies. The first case study examined the strong geomagnetic storm of 9 October 1990. The 3-day recovery phase of this storm was associated with prolonged substorm activity and gradual electron acceleration to relativistic energies, coincident with enhanced levels of whistler mode chorus. The second case study examined the strong geomagnetic storm of 26 August 1990. In contrast to case 1, the 3-day recovery phase of this storm was associated with little substorm activity, and a net reduction in the flux of relativistic electrons, coincident with reduced levels of whistler mode chorus. The third case study examined an extended period (11-16 September 1990) of prolonged substorm activity 
in the absence of a significant storm signature, and was characterised by gradual acceleration of electrons to relativistic energies and enhanced levels of whistler mode chorus. Horne et al. (2002) examined the temporal evolution of the pitch angle distributions in the energy range $0.15<E<1.58$ $\mathrm{MeV}$ during the 9 October 1990 storm, and observed energydependent flat-top distributions during the recovery phase. The foregoing results are all consistent with a local stochastic acceleration mechanism to relativistic energies, driven by substorm-enhanced whistler mode chorus.

Here we take a further detailed look at the 9 October 1990 storm. Specifically, we examine the evolution of the spectral responses of the electrons and the waves during the recovery phase, to see if the observed responses are consistent with an acceleration mechanism involving whistler mode chorus.

\subsection{Solar wind and geophysical parameters}

The solar wind and geophysical parameters for the 9 October 1990 storm are shown in Fig. 1, together with CRRES positional information and a plot of the ratio $f_{p e} / f_{c e}$. From top to bottom, the panels show the universal time, magnetic local time, magnetic latitude, the ratio $f_{p e} / f_{c e}$, solar wind speed and IMF $B_{z}$ data from IMP 8 , the colour-coded $D_{s t}$ index, and finally, the colour-coded $K_{p}$ index, together with a line plot of the $A_{E}$ index. Each orbit is defined to start and stop at perigee so that in the top four panels, one orbit of data is represented by two vertical stripes, corresponding to the outbound and inbound legs, respectively. The approximate position of the plasmapause, $L_{p}$, given by the expression:

$L_{p}=5.6-0.46 K_{p}^{*}$,

where $K_{p}^{*}$ is the maximum value of the $K_{p}$ index in the previous $24 \mathrm{~h}$ (Carpenter and Anderson, 1992), is marked on the upper four traces as a thin white line. The coloured vertical bars denote the times of apogee of the orbits used in the subsequent analysis.

The storm begins with a storm sudden commencement, as evidenced by the increase in $D_{s t}$ to $31 \mathrm{nT}$ at 14:30 UT on 9 October 1990. The main phase of this storm commences at around 00:30 UT on 10 October 1990 and lasts for approximately $8 \mathrm{~h}$. During this period the IMF has a strong southward component and the solar wind speed is approximately $500 \mathrm{~km} / \mathrm{s}$. A $D_{s t}$ minimum of $-133 \mathrm{nT}$ is reached at 08:30 UT, indicative of a strong geomagnetic storm. The main phase is then followed by a long recovery phase containing several subsidiary $D_{s t}$ minima, with the $D_{s t}$ index eventually returning to quiet-time values after approximately 3.25 days. The $A_{E}$ index is elevated during the main phase with a peak value of $970 \mathrm{nT}$ just after the $D_{s t}$ minimum. The $A_{E}$ index fluctuates, but remains predominantly enhanced during the entire 3.25-day recovery period.

\subsection{CRRES sampling}

Figure 2 shows the spatial dependence of the equatorial, lower-band chorus electric field amplitudes during disturbed
$\left(A_{E}>150 \mathrm{nT}\right)$ conditions. Superimposed on this plot is the track of the CRRES spacecraft during orbit 189, which took place in the middle of the recovery phase between 11:21 and 21:14 UT on 11 October 1990 (solid black line). The CRRES orbit during this storm is thus well placed to examine the behaviour of the waves and the particles in the outer radiation belt, since both the outbound and inbound legs pass through regions of enhanced chorus amplitudes. The CRRES observations in the region $4<L<6$ comprise two measurements per orbit at each $L$, the outbound orbital measurements occurring between 02:00 and 04:00 MLT and the inbound measurements occurring between 06:00 and 08:00 MLT. The combination of the Earth's dipole tilt and the inclination of the CRRES orbit restricts the magnetic latitude coverage of the CRRES spacecraft to the range $-30^{\circ}<\lambda_{m}<30^{\circ}$. However, since the region of enhanced high-latitude chorus is not well sampled during this storm (Meredith et al., 2001), we choose to restrict our analysis further to include only those orbital crossings which lie within approximately $15^{\circ}$ of the magnetic equator. This also has a beneficial effect on the particle analysis, since it reduces the modulating effect of changing magnetic latitude on the local perpendicular flux which occurs when the particle distribution function is anisotropic and which tends to become more apparent at higher latitudes. We make one exception, and plot the fluxes at the $D_{s t}$ minimum to show the extent of the associated flux drop out.

\subsection{Electron spectral response}

We begin by examining the spectral response of the electrons as a function of time during the geomagnetic storm. Figure 3 shows a plot of the perpendicular electron flux as a function of energy at $L=4,4.5,5$ and 6 , taken at various times throughout the event. Results are shown for both the inbound and outbound passes, and the profiles are colour-coded to identify the times of the crossings. There is an initial reduction in the perpendicular flux associated with the storm sudden commencement on 9 October, as may be seen by comparing the spectra from the outbound leg of orbit 185 with the spectra from orbit 180 . This is not a $D_{s t}$ effect, but rather due to loss via scattering at the magnetopause and/or interactions with enhanced plasma waves. Electron injections associated with the first few hours of the main phase then lead to increased fluxes of seed electrons with energies of the order of several hundred $\mathrm{keV}$, as may be seen by comparing the spectra from the inbound and outbound legs of orbit 185. This is followed by a flux drop out at all energies associated with the $D_{s t}$ effect and other non-adiabatic loss mechanisms. This is evidenced by the sharp fall in fluxes between the inbound leg of orbit 185, which takes place during the first few hours of the main phase, and the outbound leg of orbit 186, which takes place around the $D_{s t}$ minimum. The fluxes then start to recover during the inbound leg as the $D_{s t}$ index increases. By the end of orbit 186, the flux of seed electrons with energies of the order of a few hundred $\mathrm{keV}$ are enhanced over pre-storm levels. In sharp contrast, the flux of relativistic electrons remains depleted when compared to the pre-storm 


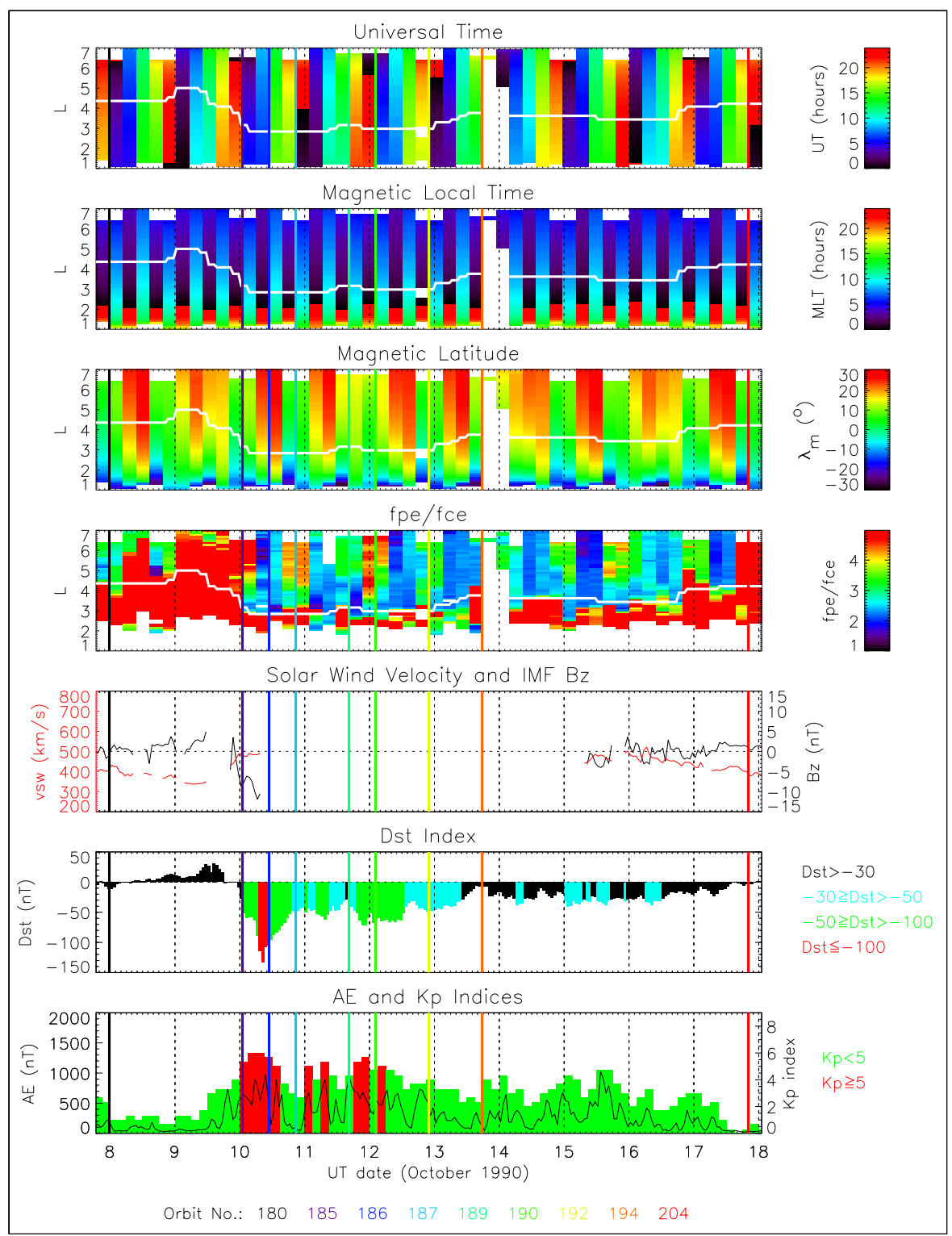

Fig. 1. Geophysical indices, solar wind parameters and the ratio $f_{p e} / f_{c e}$ for orbits 180 to 204 . From top to bottom, the panels show the universal time, magnetic local time, magnetic latitude, the ratio $f_{p e} / f_{c e}$, solar wind speed and IMF $B_{z}$ data from IMP 8 , the colour-coded $D_{s t}$ index, and the colour-coded $K_{p}$ index, together with a line plot of the $A_{E}$ index.

levels, as may be seen by comparing the spectra from the inbound legs of orbits 180 and 186. Further injections of seed electrons with energies of the order of a few hundred $\mathrm{keV}$ are also observed during the inbound leg of orbit 189 and the outbound leg of orbit 190 . The fluxes at higher energies gradually increase during the recovery phase. This is particularly apparent at $L=4$ and $L=4.5$, where the flux of electrons greater than approximately $500 \mathrm{keV}$ gradually increases during the recovery phase. The same trend of increasing fluxes at higher energies is also apparent at $L=5$ and $L=6$. However, the trend here is not so clear, and may be influenced by competing mechanisms, such as losses at the magnetopause. At relativistic energies, the largest fluxes at each $L$ are seen at the end of the event when the $D_{s t}$ index has returned to approximately $0 \mathrm{nT}$. The overall flux increase is of the order of a factor of 10 for the $1.58 \mathrm{MeV}$ electrons at $L=4$ and $L=4.5$. At $L=5$ and $L=6$, the flux increases at $E=1.58 \mathrm{MeV}$ are of the order of 5 and 3, respectively.

As lower energy electrons are injected into the outer radiation zone during substorm activity, their flux below a few hundred $\mathrm{keV}$ remains remarkably stable throughout the storm recovery phase (orbits 186-204). The phase space density of such electrons is also well below that of the source population in the near-Earth plasma sheet. The spectral characteristics of electrons below a few hundred $\mathrm{keV}$ are consistent with the concept of stable trapping with a limiting differential spectrum $J^{*}(E) \sim 1 / E$, as proposed by Schulz and Davidson (1988), based on the original concept devised by 


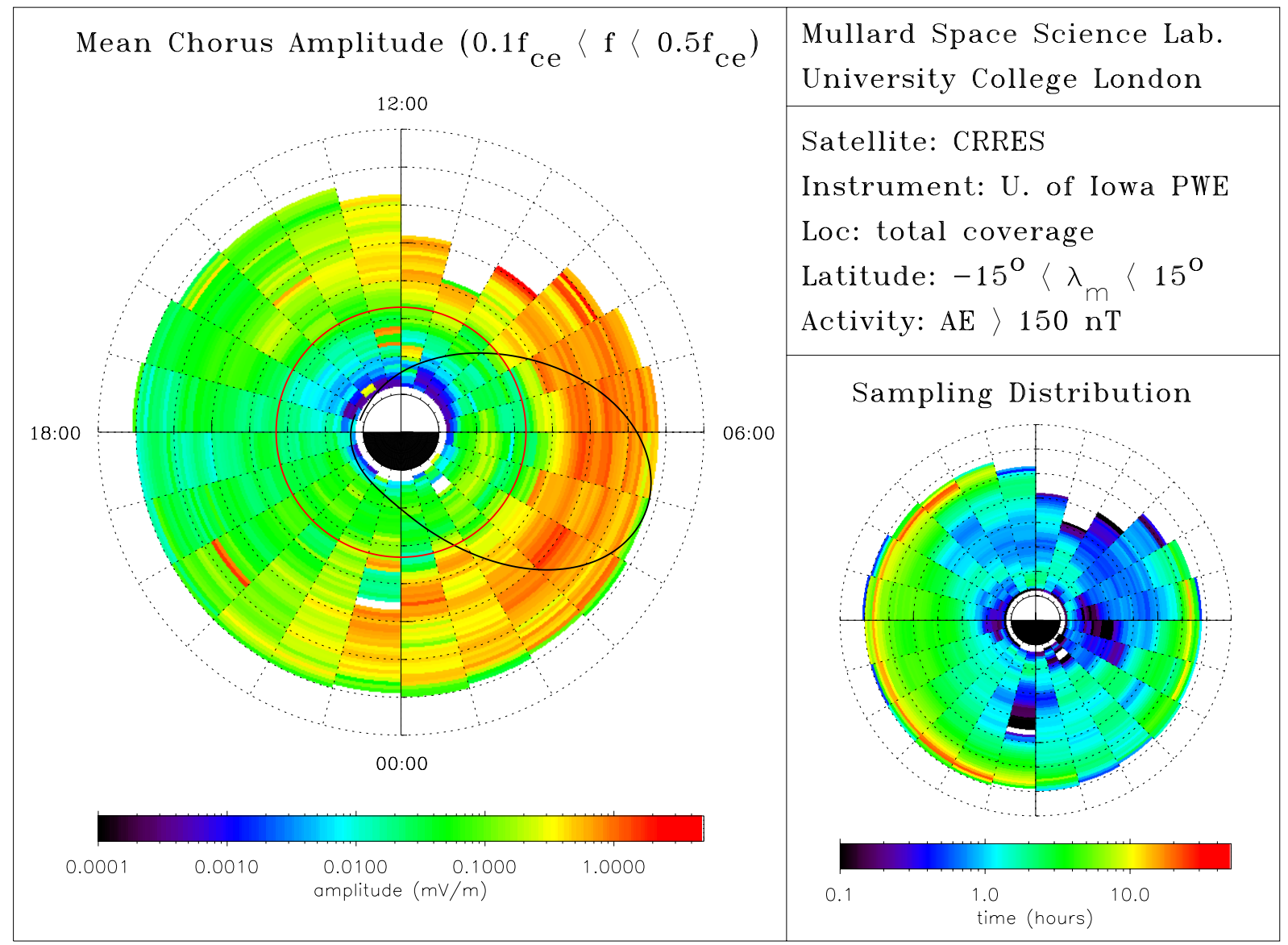

Fig. 2. Average equatorial lower-band chorus electric field amplitudes as a function of $L$ and MLT during disturbed $\left(A_{E}>150 \mathrm{nT}\right)$ conditions. The solid black line represents the track of CRRES during orbit 189. The solid red line shows the empirical position of the plasmapause for $K_{p}^{*}=5$.

Kennel and Petschek (1966). This suggests that electrons up to a few hundred $\mathrm{keV}$ are the source population for excitation of whistler mode waves which rapidly scatter electrons into the atmosphere and limits their flux below $J^{*}(E)$, regardless of the intensity of the substorm injection process. The absolute levels of the self-limiting flux are comparable to those reported by Davidson et al. (1988) near $L=6$, and show the expected $J^{*} \sim 1 / L^{4}$ scaling at lower $L$.

Brautigam and Albert (2000) performed a detailed phase space density analysis on the 9 October 1990 geomagnetic storm, using data from the CRRES spacecraft. They demonstrated that the temporal variability of the lower energy electrons, with first adiabatic invariant, $M\left(=p_{\perp}^{2} / 2 m_{e} B\right)<$ $314 \mathrm{MeV} / \mathrm{G}$, could be explained purely in terms of variations at the outer boundary ( $L=6.6)$ and $K_{p}$-dependent radial diffusion. However, the higher energy electrons with $M>\sim 700 \mathrm{MeV} / \mathrm{G}$ exhibited a decrease in phase space density with increasing $L$, which is inconsistent with a source driven by inward radial diffusion.

We can use the results of Brautigam and Albert (2000) to calculate the energy spectrum predicted by their radial diffusion model to investigate whether this is consistent with the observed spectral response. We begin by converting the first adiabatic invariant to energy at $L=4.5$, using Fig. 3 of Brautigam and Albert (2000). The normalised phase space densities, $f_{n}=m_{e}^{3} J / p^{2}$, used in the Brautigam and Albert (2000) paper are read directly from their Fig. 7, and then converted to differential number flux, $J$. The results are plotted in Fig. 4 for the nominal diffusion model (green trace), the upper and lower bounds of the model (yellow and red traces, respectively), together with the observed fluxes at the beginning (black trace) and end (blue trace) of the event. If we compare the model curve with the observed differential number fluxes at the end of the event, we find that the model gives a good estimate of the fluxes seen at energies $E<\sim$ $0.8 \mathrm{MeV}$. However, at higher energies, $E>\sim 0.8 \mathrm{MeV}$, the model underestimates the flux at $L=4.5$. We conclude that standard radial diffusion cannot reproduce the high-energy tail seen in the data. The radial diffusion coefficients would need energy-dependence with larger diffusion rates at higher energies or alternatively greater loss rates at lower energies. Neither of these effects are included in current radial diffusion modelling.

We now consider whether the energy-dependence in the 


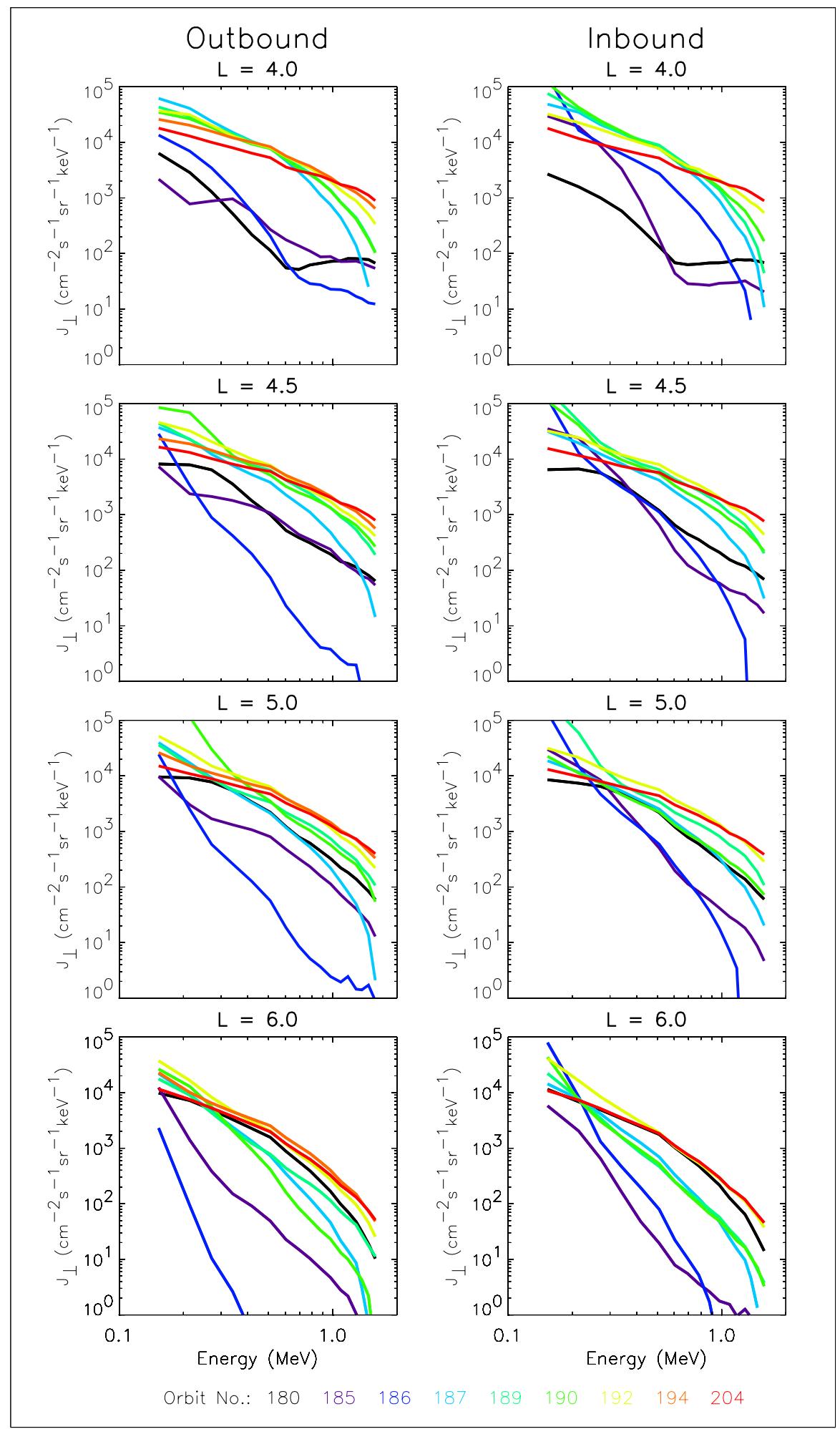

Fig. 3. The perpendicular electron flux as a function of energy at $L=4,4.5,5$ and 6 , taken at various times throughout the event. Results are shown for both the inbound and outbound passes, and the profiles are colour-coded to identify the times of the crossings.

high-energy spectrum is consistent with electron resonance, with whistler mode waves as evidence of wave-particle interactions playing an important role in the acceleration process.

\subsection{Chorus activity and chorus spectral response}

The average equatorial $\left(\left|\lambda_{m}\right|<15^{\circ}\right)$, lower-band $\left(0.1 f_{c e}<\right.$ $f<0.5_{c e}$ ) chorus amplitudes measured by CRRES dur- 


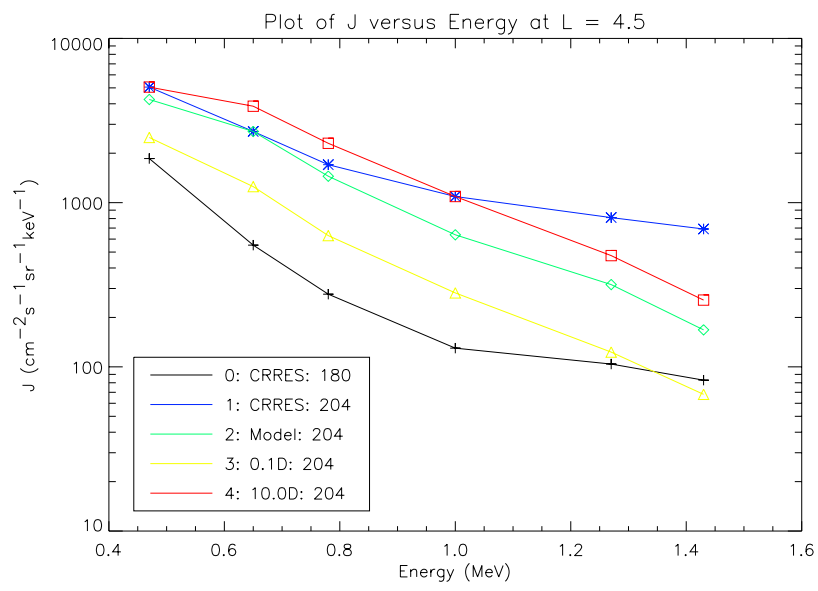

Fig. 4. The electron differential number flux as a function of energy for orbits 180 and 204, together with the results of the Brautigam and Albert (2000) radial diffusion model.

ing the 3-day recovery phase are $1.1,1.1$ and $0.8 \mathrm{mV} / \mathrm{m}$ at $L=4,5$ and 6 , respectively. These enhanced amplitudes are more than an order of magnitude larger than those typically observed during quiet periods when $A_{E}<100 \mathrm{nT}$ (Meredith et al., 2001). Since the rate of diffusion depends on the wave power, resonant electrons in this region will consequently experience an increase in the rate of velocity space diffusion by more than two orders of magnitude during the recovery phase.

During the recovery period the $A_{E}$ index is greater than $150 \mathrm{nT}$ for approximately $85 \%$ of the time. The magnitude and global extent of the whistler mode chorus during this period may be estimated using an extension of the results from our previous statistical survey. Here we adopt a similar approach to that described in Meredith et al. (2001), and determine the average chorus amplitudes as a function of spatial location for $A_{E}>150 \mathrm{nT}$. We regard this distribution as being representative of the extent and magnitude of the chorus amplitudes during the recovery phase, since the $A_{E}$ index lies in this range for $85 \%$ of the time during this period. The results of this analysis are shown in Fig. 2 for the equatorial lower-band chorus. The empirical position of the postmidnight plasmapause for $K_{p}^{*}=5$ is shown as a solid red line, confirming that the enhanced chorus amplitudes tend to lie outside of the storm-time plasmapause. This figure suggests that, during the recovery period, the equatorial lowerband chorus amplitudes will be enhanced with amplitudes typically $>0.5 \mathrm{mV} / \mathrm{m}$ over a wide range of geospace from 23:00 MLT through dawn to 13:00 MLT, over a range of Lshells from $4<L<7$. The high-latitude $\left(\left|\lambda_{m}\right|>15^{\circ}\right)$ lower-band chorus will also be enhanced during this period and, in particular, in the region 06:00-15:00 MLT (see Fig. 4 of Meredith et al., 2001). These enhanced chorus amplitudes can contribute to the acceleration of relativistic electrons.

During the storm, the cross-tail electric potential is enhanced and may reach a few hundred $\mathrm{keV}$, but it is not sufficient to accelerate electrons to $\mathrm{MeV}$ energies or to account for the increase in flux to values greater than the pre-storm level. However, the enhanced convection electric field may provide a seed population with energies of the order of a few hundred $\mathrm{keV}$, which are then subsequently accelerated to relativistic energies (see, for example, Obara et al., 2000). An equatorially mirroring electron, with an energy of $200 \mathrm{keV}$, drifts around the Earth in $64 \mathrm{~min}$ at $L=4$ and $43 \mathrm{~min}$ at $L=6$, under the influence of gradient drifts (Walt, 1994). Thus, seed electrons in the outer zone will complete orbits around the Earth on a time scale of the order of $1 \mathrm{~h}$. The seed electrons have ample opportunity to interact with the enhanced whistler mode chorus waves, since they spend approximately $60 \%$ of their orbit in regions where the equatorial lower-band chorus is enhanced and approximately $40 \%$ of their orbit in regions where the high-latitude lower-band chorus is enhanced.

We now examine the temporal response of the spectral wave intensities during the recovery phase of the storm. Figure 5 shows a plot of the intensities as a function of relative frequency, $x=f / f_{c e}$, at $L=4,4.5,5$ and 6 for the near-equatorial orbits during the recovery phase. During extended quiet periods, when the $A_{E}$ index is less than $100 \mathrm{nT}$, the wave spectral intensity typically falls below $10^{-13} V^{2} \mathrm{~m}^{-2} \mathrm{~Hz}^{-1}$. Here the quietest period occurs at the end of the event during orbit 204. The intensities are below $10^{-13} V^{2} \mathrm{~m}^{-2} \mathrm{~Hz}^{-1}$ over a wide range of frequencies for both the outbound and inbound orbits at each $L$. Major enhancements are seen during the entire recovery phase. During these periods, the chorus intensities are elevated at all $L$, and this is due to the enhanced substorm activity. The intensities maximise over a wide range of frequencies, but predominantly in the range $0.1<x<0.6$.

4.5 Resonance curves and resonant diffusion curves for gyroresonant wave-particle interactions

The results presented so far have shown that the whistler mode chorus activity is enhanced during the period of spectral hardening. However, can these waves resonate with electrons in the appropriate energy range and hence, be a viable candidate for the acceleration mechanism? To examine this question, we calculate the resonance curves and resonant diffusion curves for electron cyclotron resonance with whistler mode waves travelling parallel and anti-parallel to the magnetic field. The resonance condition for electron interaction with whistler mode waves propagating parallel to the magnetic field (see, for example, Summers et al., 1998) may be written in the form:

$x-\frac{k v_{\|}}{2 \pi f_{c e}}=\frac{1}{\gamma}$.

Here, $x$ is the relative frequency, $k$ is the wave number in the direction of propagation, $f_{c e}=e B_{0} / 2 \pi m_{e}$ is the electron gyrofrequency, $B_{0}$ is the magnitude of the ambient magnetic field, $m_{e}$ is the electron rest mass, $\gamma=1 / \sqrt{\left(1-v^{2} / c^{2}\right)}$ and $v^{2}=v_{\perp}^{2}+v_{\|}^{2}$, where $v_{\perp}$ and $v_{\|}$are the electron velocity components perpendicular and parallel to the field, respec- 


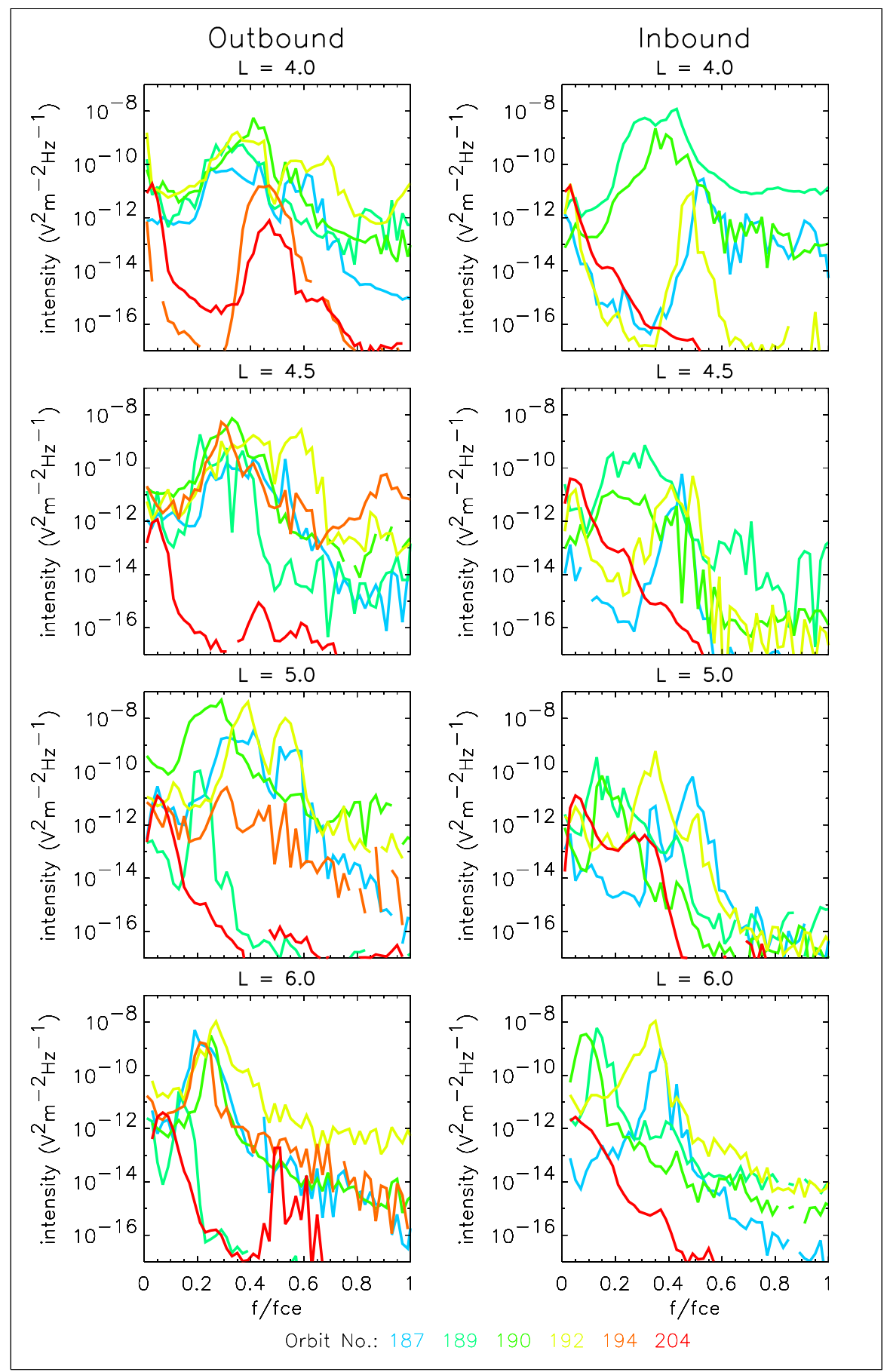

Fig. 5. The wave spectral intensities as a function of relative frequency, $x=f / f_{c e}$, at $L=4,4.5,5$ and 6 during the recovery phase.

tively. Equation (5) describes the curves in velocity (or momentum) space for which electrons are in resonance with a wave of a given frequency. Summers et al. (1998) derived the solution in parametric form for the resonant diffusion curves for electron cyclotron resonance with field-aligned whistler mode waves, using a fully relativistic treatment. These curves represent the trajectories along which electrons will move when subject to whistler mode waves over an ap- propriate broad frequency range. During gyroresonant interactions with whistler mode chorus waves, the electrons diffuse from regions of high phase space density to regions of low phase space density along the resonant diffusion curves. If this is in the direction of decreasing energy, then the electrons will lose energy and the waves will gain energy, which leads to wave growth. Conversely, if this is in the direction of increasing energy, then the electrons will gain energy at 


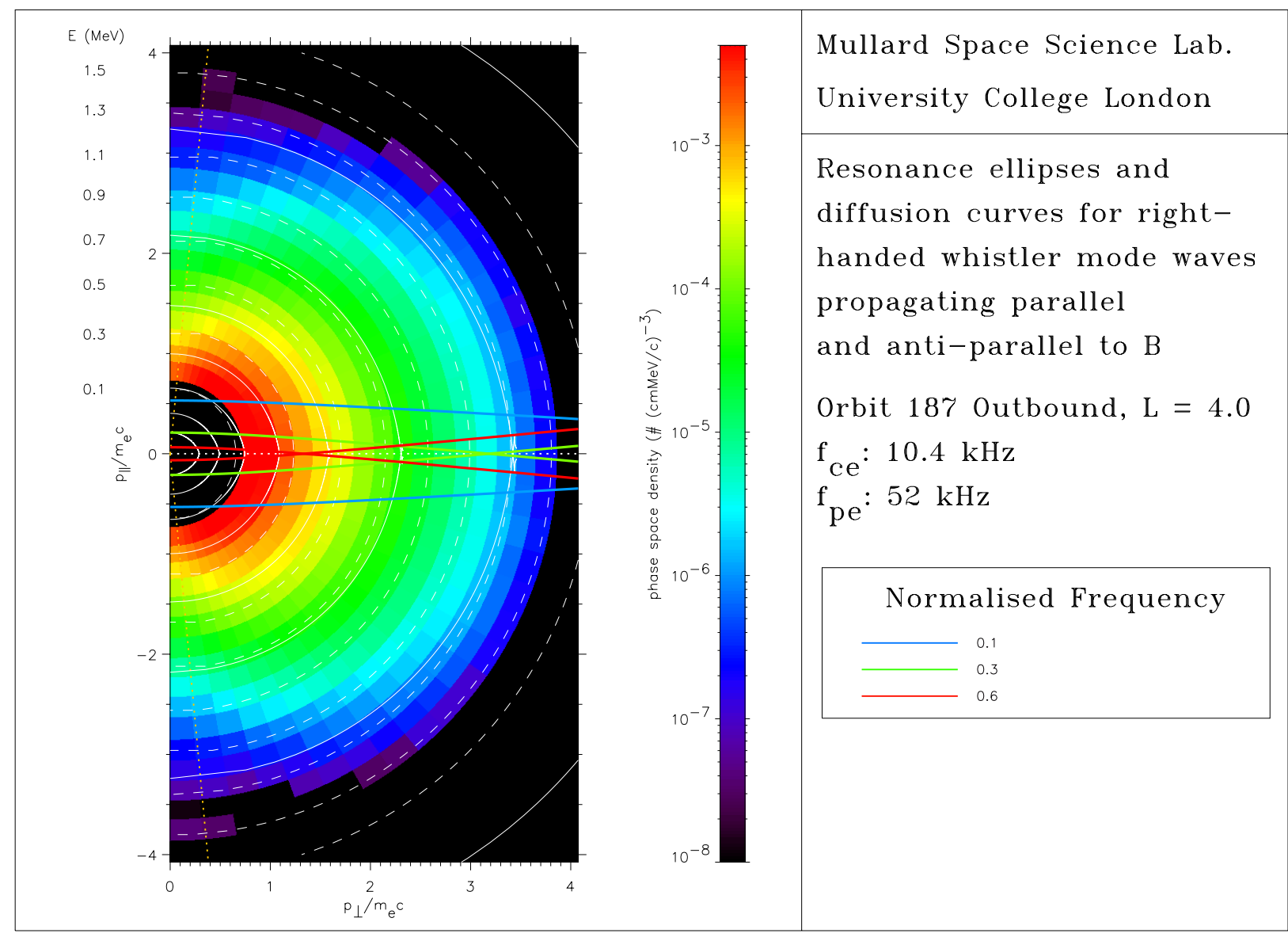

Fig. 6. The electron phase space density as a function of normalised momentum, parallel and perpendicular to the magnetic field. The $90^{\circ}$ pitch angle is marked by the white dotted line, and the edge of the loss cone, by the yellow dotted line. Contours of constant energy are plotted as dashed lines and labelled on the left-hand side. Examples of the resonance curves for the case $f_{p e}=5 f_{c e}$ are plotted, and colour-coded for three distinct frequencies in the range $0.1<x<0.6$, and examples of the resonant diffusion curves are plotted as solid white lines.

the expense of the waves.

We assume that whistler mode chorus waves are enhanced over a broad range of frequencies in the range $0.1<x<0.6$. A critical parameter in determining the resonance curves and the resonant diffusion curves is the ratio of the electron plasma frequency to the electron gyrofrequency. This parameter is plotted as a function of half-orbit and $L$ in steps of $0.1 \mathrm{~L}$ in the central panel of Fig. 1. Outside of the plasmapause during the recovery phase of this storm, the ratio $f_{p e} / f_{c e}$ is variable but typically lies in the range $2 \leq f_{p e} / f_{c e} \leq 5$ near the equator. At higher latitudes, $f_{p e} / f_{c e}$ may fall to values close to unity. Also, estimates of $f_{p e}$ from the lower frequency cutoff may give an overestimate, as density irregularities may scatter and reflect continuum radiation thereby preventing the lower frequency component from reaching the spacecraft. Therefore, we select $f_{p e} / f_{c e}=1$ and 5 as being representative of the range of values outside of the plasmapause during the storm recovery phase.

A representative plot of the electron phase space density at $L=4$ during the storm recovery phase is shown in Fig. 6 .
The electron phase space density is plotted as a function of normalised momentum, parallel and perpendicular to the magnetic field. The $90^{\circ}$ pitch angle is marked by the white dotted line, and the edge of the loss cone, which, assuming a dipole field, is $5.3^{\circ}$ wide at $L=4$, is marked as the yellow dotted line. Contours of constant energy are plotted as dashed lines. Examples of the resonance curves given by Eq. (5) for the case $f_{p e}=5 f_{c e}$ are plotted and colour-coded for three distinct frequencies in the range $0.1<x<0.6$, and examples of the resonant diffusion curves (using the results of Summers et al., 1998) for this case are plotted as solid white lines. Waves in the frequency range $0.1<x<0.6$ are resonant with electrons over a limited region of momentum space, principally in the region $\left|p_{\|} / m_{e} c\right|<\sim 0.5$. Moreover, the resonant diffusion curves are roughly circular, which implies limited energy diffusion and ineffective local electron acceleration in these circumstances.

In regions where $f_{p e} \approx f_{c e}$, energy diffusion may be more effective (Summers et al., 1998). The resonant diffusion curves for this case (Fig. 7) are more anisotropic and 


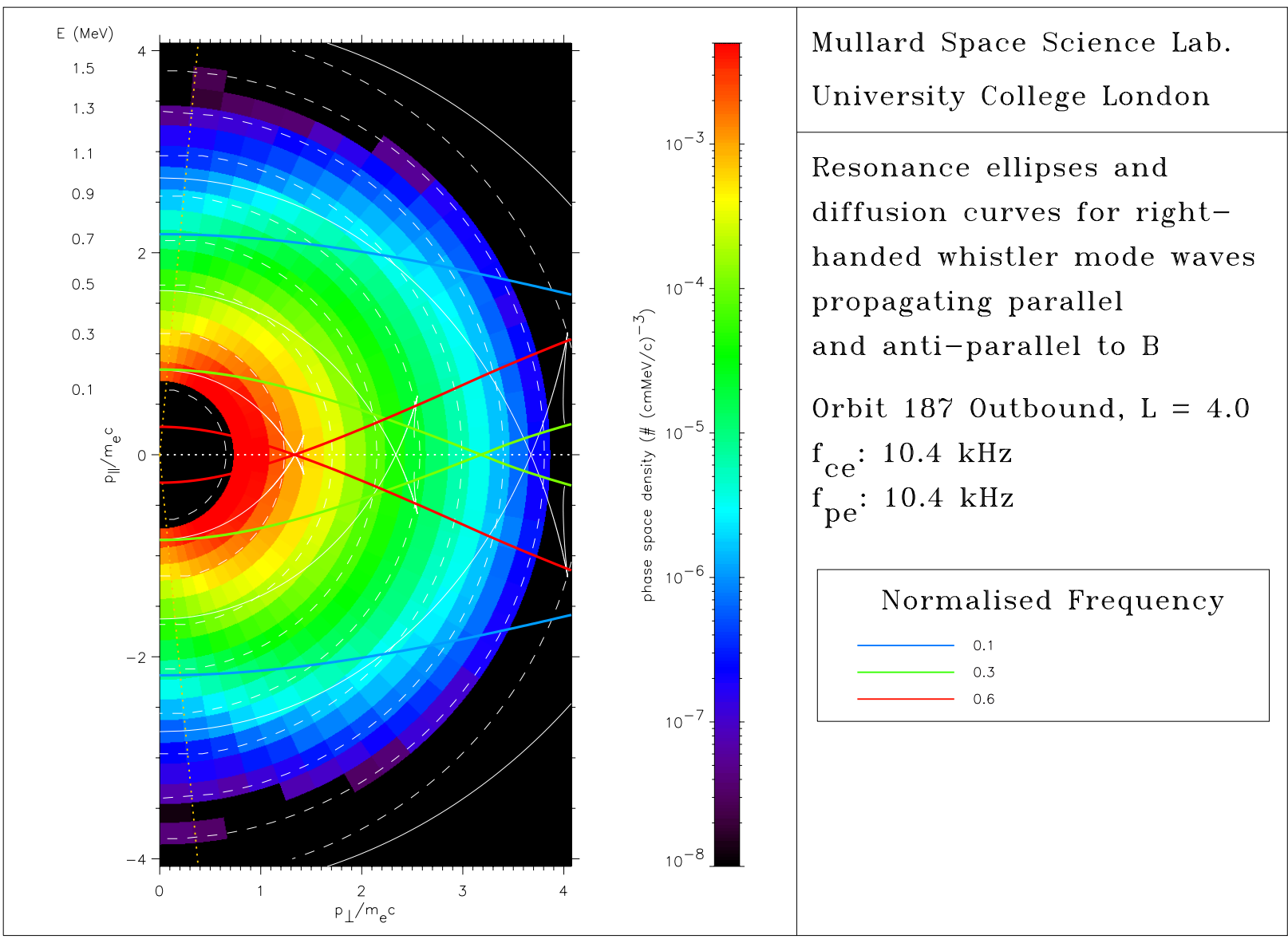

Fig. 7. The electron phase space density as a function of normalised momentum in the same format as Fig. 6. The resonance curves and the resonant diffusion curves for the case $f_{p e}=f_{c e}$ are superposed. The resonant diffusion curves for this case are more anistropic and indicate a greater potential for energy diffusion. Furthermore, the waves in the frequency range $0.1<x<0.6$ are able to resonate with electrons over a much larger region of momentum space.

have a greater potential for energy diffusion. Moreover, the waves in the frequency range $0.1<x<0.6$ are able to resonate with electrons over a much larger region of momentum space. Close to the loss cone, waves in this frequency range are able to resonate with electrons in the energy range $20 \mathrm{keV}<E<700 \mathrm{keV}$. On the other hand, close to the $90^{\circ}$ pitch angle, these waves are able to resonate with electrons with energies in the energy range $340 \mathrm{keV}<E<4.6 \mathrm{MeV}$. Thus, close to the $90^{\circ}$ pitch angle we anticipate a minimum energy threshold for perpendicular energisation (given by the intersection point of the two red lines) by gyroresonant waveparticle interactions with a given band of whistler mode chorus.

Resonant electrons close to the loss cone will diffuse along the resonant diffusion curves in the direction of decreasing phase space density, which, in this region, is towards the loss cone and in the direction of decreasing energy. These electrons will lose energy and contribute to wave growth. For example, whistler mode waves with $x=0.3$ interact with $\left|p / m_{e} c\right|=0.84(E=160 \mathrm{keV})$ electrons near the loss cone. Moving away from the loss cone, the same frequency waves interact with higher energy electrons. For example, at pitch angles of $45^{\circ}$ and $90^{\circ}$, these waves resonate with $\left|p / m_{e} c\right|=1.1(E=250 \mathrm{keV})$ electrons and $\left|p / m_{e} c\right|=3.2$ $(E=1.2 \mathrm{MeV})$ electrons, respectively. For electrons with pitch angles in the approximate range $45^{\circ}<\alpha<135^{\circ}$, the gradient in particle phase space density will result in resonant diffusion (along the solid white curves) towards the $90^{\circ}$ pitch angle and higher energy. Such electrons will consequently be energised by gyroresonant interactions with the enhanced chorus waves, and their energisation will contribute towards wave damping. It is important to realise that even though electron acceleration is occurring for the high energy population at larger pitch angles, waves can still experience net wave growth due to the much larger flux of lower energy resonant electrons near the loss cone.

We have considered two extreme cases for the ratio $f_{p e} / f_{c e}$. However, in the course of the drift of a particle around the Earth, different regions of geospace will be encountered, and the particle may experience a wide range of conditions. The shape of the resonance curves and resonant diffusion curves depends on the ratio $f_{p e} / f_{c e}$. Energy diffu- 
sion will be most effective in regions of lower density and, in particular, in regions where $f_{p e} / f_{c e} \sim 1$. In these regions, the observed chorus waves can resonate with electrons over a wide range of energies, including the important energy range from $100 \mathrm{keV}$ to several $\mathrm{MeV}$. These waves can drive electrons to higher energies along the resonant diffusion curves, and hence, energise the electrons. Summers et al. (1998) proposed a model for the gradual acceleration of electrons to relativistic energies during the recovery phase of geomagnetic storms, which involved a combination of energy diffusion by enhanced whistler mode waves outside of the plasmasphere and pitch angle scattering by enhanced EMIC waves near the dusk-side plasmapause. In higher density regions, the resonant diffusion curves become more isotropic, and hence, gyroresonant interactions with enhanced whistler mode chorus in these regions may also contribute to the pitch angle scattering invoked in this model. We also anticipate a minimum perpendicular energy for energisation that will be dependent on the upper frequency limit of the enhanced chorus emissions encountered by the electrons as they drift around the Earth. This is seen in particle observations that which show an energy-dependent response. Our results are consistent with the theory of electron stochastic acceleration to relativistic energies, driven by gyroresonant wave-particle interactions with whistler mode chorus waves, and suggest that these waves can provide an internal source of energetic electrons during the recovery phase of this geomagnetic storm that radial diffusion cannot.

\section{Conclusions}

We have used wave and particle data from the Combined Release and Radiation Effects Satellite (CRRES) to study the role of wave-particle interactions in the generation of relativistic electrons during storms. We have examined the temporal behaviour of the spectral response of the electrons and waves during the 9 October 1990 geomagnetic storm. The main conclusions are as follows:

1. The observed hardening of the electron energy spectra over about 3 days during the recovery phase is coincident with prolonged substorm activity, as monitored by the $A_{E}$ index, and enhanced levels of whistler mode chorus waves.

2. The theory of gyroresonant interaction with whistler mode waves predicts a minimum energy threshold for perpendicular energisation, which is consistent with the observed energy-dependent spectral response of the electrons.

3. The spectral hardening is observed to take place over a range of energies appropriate to the resonant energies associated with Doppler-shifted cyclotron resonance, as supported by the construction of realistic resonance curves and resonant diffusion curves.
4. The observed spectral hardening is not consistent with energy-independent radial diffusion models.

5. The results in this paper provide strong circumstantial evidence for the local stochastic acceleration of electrons to relativistic energies, driven by wave-particle interactions involving whistler mode chorus.

6. An additional significant result of this paper is that we demonstrate that the lower energy part of the storm-time electron distribution is in steady-state balance, in accordance with the Kennel and Petschek (1966) theory of limiting stably-trapped particle fluxes.

Acknowledgements. We thank A. Vampola for providing the CRRES MEA data used in this study and for his assistance in their analysis and interpretation. We also thank the World Data Centre C1 for STP at the Rutherford Appleton Laboratory and the NSSDC Omniweb for providing the geomagnetic indices and solar wind parameters used in this paper. D. S. acknowledges support from the Natural Sciences and Engineering Research Council of Canada under grant A-0621. Additional support is acknowledged from NSF grant ATM 9729021 and NASA grant NAG5 4680.

Topical Editor G. Chanteur thanks J. Albert and another referee for their help in evaluating this paper.

\section{References}

Anderson, R. R., Gurnett, D. A., and Odem, D. L.: CRRES Plasma Wave Experiment, J. Spacecr. Rockets, 29, 570, 1992.

Baker, D. N.: An assessment of the space environmental conditions during the recent Anik E1 spacecraft operational failure, ISTP Newsl., 6, 2, 8, 1996.

Baker, D. N., Blake, J. B., Callis, L. B., Belian, R. D., and Cayton, T. E.: Relativistic electrons near geostationary orbit: Evidence for internal magnetospheric acceleration, Geophys. Res. Lett, 16, 559, 1989.

Baker, D. N., Kanekal, S. G., Looper, M. D., Blake, J. B., and Mewaldt, R. A.: Jovian, solar and other possible sources of radiation belt particles, in: Radiation Belts: Models and Standards, (Eds) Lemaire, J. F., Heynderickx, D., and Baker, D. N., Geophys. Monogr. Ser., 97, 49, AGU, Washington D. C., 1996.

Baker, D. N., Li, X., Turner, N., Allen, J. H., Bargatze, L. F., Blake, J. B., Sheldon, R. B., Spence, H. E., Belian, R. D., Reeves, G. D., Kanekal, S. G., Klecker, B., Lepping, R. P., Olgilvie, K., Mewaldt, R. A., Onsager, T., Singer, H. J., and Rostoker, G.: Recurrent geomagnetic storms and relativistic electron enhancements in the outer magnetosphere: ISTP coordinated measurements, J. Geophys. Res., 102, 14, 141, 1997.

Beutier, T. and Boscher, D.: A 3-dimensional analysis of the electron radiation belt by the Salammbô code, J. Geophys. Res., 100, 14, 853, 1995.

Blake, J. B., Selesnick, R. S., Fennel, J. F., Grande, M., and Perry, C. H.: A comparison of the injection parameters of relativistic electrons and ring current ions as observed by CRRES, EOS Trans. AGU, 79, 24, W99, 1998.

Brautigam, D. H. and Albert, J. M.: Radial diffusion analysis of outer radiation belt electrons during the 9 October 1990, magnetic storm, J. Geophys. Res., 105, 291, 2000. 
Carpenter, D. L. and Anderson, R. R.: An ISEE/whistler model of equatorial electron density in the magnetosphere, J. Geophys. Res., 97, 1097, 1992.

Davidson, G. T., Filbert, P. C., Nightingale, R. W., Imhof, W. L., Reagan, J. B., and Whipple, E. C.: Observations of intense trapped electron fluxes at synchronous altitudes, J. Geophys. Res., 93, 77, 1988.

Elkington, S. R., Hudson, M. K., and Chan, A. A.: Acceleration of relativistic electrons via drift-resonance interaction with toroidalmode Pc5 ULF oscillations, Geophys. Res. Lett., 26, 3273, 1999.

Fok, M. C., Moore, T. E., and Spjeldvik, W. N.: Rapid enhancement of radiation belt electron fluxes due to substorm dipolarization of the geomagnetic field, J. Geophys. Res., 106, 3873, 2001.

Fujimoto, M. and Nishida, A.: Energization and anisotropization of energetic electrons in the Earth's radiation belt by the recirculation process, J. Geophys. Res., 95, 4265, 1990.

Gurnett, D. A. and Shaw, R. R.: Electromagnetic radiation trapped in the magnetosphere above the plasma frequency, J. Geophys. Res., 78, 8136, 1973.

Horne, R. B. and Thorne, R. M.: Potential waves for relativistic electron scattering and stochastic acceleration during magnetic storms, Geophys. Res. Lett., 25, 3011, 1998.

Horne, R. B., Meredith, N. P., Thorne, R. M., Heynderickx, D., Iles, R. H. A., and Anderson, R. R.: Evolution of energetic elecron pitch angle distributions during storm time electron acceleration to MeV energies, J. Geophys. Res., submitted, 2002.

Hudson, M. K., Elkington, S. R., Lyon, J. G., and Goodrich, C. C.: Increase in relativistic electron flux in the inner magnetosphere: ULF wave mode structure, Adv. Space Res., 25, 2327, 2000.

Iles, R. H. A., Fazakerley, A. N., Johnstone, A. D., Meredith, N. P., and Bühler, P.: The relativistic electron response in the outer radiation belt during magnetic storms, Ann. Geophysicae, this issue, 2002.

Kennel, C. F. and Petschek, H. E.: Limit on stably trapped particle fluxes, J. Geophys. Res., 71, 1, 1966.

Kim, H. J., Chan, A. A., Wolf, R. A., and Birn, J.: Can substorms produce relativistic outer belt electrons?, J. Geophys. Res., 105, $7721,2000$.

Koons, H. C. and Roeder, J. L.: A survey of equatorial magnetospheric wave activity between 5 and $8 R_{E}$, Planet. Space Sci., $38,1335,1990$

Li, X. and Temerin, M. A.: The electron radiation belt, Space Sci. Rev., 95, 569, 2001.

Li, X., Baker, D. N., Temerin, M., Cayton, T. E., Reeves, G. D., Selesnick, R. S., Blake, J. B., Lu, G., Kanekal, S. G., and Singer, H. J.: Rapid enhancements of relativistic electrons deep in the magnetosphere during the 15 May 1997, magnetic storm, J. Geophys. Res., 104, 4467, 1999.

Liu, W. W., Rostoker, G., and Baker, D. N.: Internal acceleration of relativistic electrons by large-amplitude ULF pulsations, J. Geophys. Res., 104, 17 391, 1999.

Mathie, R. A. and Mann, I. R.: A correlation between extended intervals of ULF wave power and storm-time geosynchronous relativistic electron flux enhancements, Geophys. Res. Lett., 27, $3261,2000$.

Meredith, N. P., Horne, R. B., and Anderson, R. R.: Substorm dependence of chorus amplitudes: Implications for the acceleration of electrons to relativistic energies, J. Geophys. Res., 106, $13165,2001$.

Meredith, N. P., Horne, R. B., Iles, R. H. A., Thorne, R. M., Heynderickx, D., and Anderson, R. R.: Outer zone relativistic electron acceleration associated with substorm enhanced whistler mode chorus, J. Geophys. Res., in press, 2002.

Obara, T., Nagatsuma, T., Den, M., Miyoshi, Y., and Morioka, A.: Main phase creation of "seed" electrons in the outer radiation belt, Earth Planets Space, 52, 41, 2000.

O’Brien, T. P., McPherron, R. L., Sornette, D., Reeves, G. D., Friedel, R., and Singer, H. J.: Which magnetic storms produce relativistic electrons at geosynchronous orbit?, J. Geophys. Res., 106, 15533,2001

Roth, I., Temerin, M., and Hudson, M. K.: Resonant enhancement of relativistic electron fluxes during geomagnetically active periods, Ann. Geophysicae, 17, 631, 1999.

Schulz, M. and Davidson, G. T.: Limited energy spectrum of a saturated radiation belt, J. Geophys. Res., 93, 59, 1988.

Schulz, M. and Lanzerotti, L. J.: Particle Diffusion in the Radiation Belts, Springer-Verlag, 1974.

Singer, H. J., Sullivan, W. P., Anderson, P., Mozer, F., Harvey, P., Wygant, J., and McNeil, W.: Fluxgate Magnetometer Instrument on the CRRES, J. Spacecr. Rockets, 29, 599, 1992.

Summers, D. and Ma, C. Y.: A model for generating relativistic electrons in the Earth's inner magnetosphere based on gyroresonant wave-particle interactions, J. Geophys. Res., 105, 2625 , 2000.

Summers, D., Thorne, R. M., and Xiao, F.: Relativistic theory of wave-particle resonant diffusion with application to electron acceleration in the magnetosphere, J. Geophys. Res., 103, 20487 , 1998

Vampola, A. L., Osborn, J. V., and Johnson, B. M.: CRRES Magnetic Electron Spectrometer, J. Spacecr. Rockets, 29, 592, 1992.

Walt, M.: Introduction to Geomagnetically Trapped Radiation, Cambridge University Press, 1994. 